\title{
Comparison of Communication Protocol Power Consumption of Fine-Dust IoT Sensor Based on oneM2M Standard Protocol
}

\author{
Seong-Se Cho ${ }^{1}$, Seung-Hun $\mathrm{Kim}^{2}$ and Won-Hyuck Choi ${ }^{3 *}$ \\ ${ }^{1,2}$ Department of Aeronautical Systems Engineering Hanseo University, 236-49, \\ Gomseom-ro, Nam-myeon, Taean-gun, 32158 Chungcheongnam-do, Korea \\ ${ }^{3}$ Department of Avionics Engineering Hanseo University, 236-49, Gomseom-ro, Nam- \\ myeon, Taean-gun, 32158 Chungcheongnam-do, Korea \\ 1'choiwh@hanseo.ac.kr, ${ }^{2}$ kimsh014@gmail.com, ${ }^{3}$ chosse63@gmail.com
}

\begin{abstract}
As everything is connected to the Internet, the Internet of Things can communicate without user intervention. The Internet of Things has recently been used in various areas such as smart homes, smart cars, smart factories, and industrial sites. Manufacturing, Agriculture, and we built a system that communicates between client servers using the communication protocols MQTT and CoAP communication protocols with fine dust sensors in an HTTP communication protocol environment and identified the amount of power used for each communication.
\end{abstract}

Keywords: One M2M, MQTT, CoAP, Standard protocol, Fine dust, IoT

\section{Introduction}

Everything is connected to the Internet, allowing interaction through communication between objects without user intervention, and the Internet of Things has recently been farming, construction, smart homes, smart cars, smart factories, manufacturing, etc. We focus on various fields such as other industrial sites [1].

According to market research agencies and experts, the size of the relevant IoT market is expected to expand in 2020 and connect 50 billion Internet devices of the world. In order to take the initiative in the IoT ecosystem, labor is undertaken to anticipate patents and standards through intense technological development. Things use sensors to identify various behaviors and changes in the environment surrounding what we do in daily life and social activities and send them as digital data over the Internet. This means that digital copies of the real world are created in the cyber world or in the cloud. As the number of objects connected to the Internet increases, more accurate digital copies of the real world are created.

The IoT industry can be categorized into platforms, services, networks, security and devices [2].

First, in the platform area, global giants such as Google, Microsoft, and SKT are trying to become leaders in the IoT ecosystem, but there are no operators or SMEs that still dominate the IoT platform. Hesitate to enter the market and enter the market. The service sector is changing from the previous environment of using specific handsets to an open ecosystem where anyone can develop and use smartphones [3][4].

Article history:

Received (March 31, 2019), Review Result (July 7, 2019), Accepted (November 22, 2019) 
Because the resources of devices that use IoT are limited, the resources of IoT devices are small and the communication environment is limited, so long-term use is required. Communication of such devices requires optical communication protocols, and protocols such as MQTT and CoAP that can be used in IoT are attracting attention. I want to compare the efficiency of the two protocols used in IoT [8].

In this document, MQTT and CoAP are used to build a communication environment between devices, for the Internet of Things and for fine dust sensors in the oneM2M platform using Arduino, focusing on the power ratio (HTTP) of open source hardware consumed by each communication protocol.

\section{Implementation}

Using the Arduino IDE development tool, Ryan developed a communication program using CoAP, MQTT, and HTTP protocols on the ATmega328 board. The client uses the external power to verify the power used every 30 seconds and uses each communication protocol to transmit the measured amount of power to the server. Voltage and current amounts were measured to check the external power supply [5][9].

The system used Wi-Fi, a typical solution for TCP/IP systems, based on the IEEE 802.15.4 standard and oriented toward low power, low-speed communication, and low cost. In addition, by deploying the system over Wi-Fi-based wireless sensor network, the MQTT protocol can be run independently at the network layer without relying on the UCP protocol and is seen as a suitable protocol for message delivery service in the Internet of Things environment based on various wired and wireless network infrastructure. A wireless sensor network consists of multiple nodes, and when connecting all nodes with one hop, the network is scalable and there is a limit to connecting multiple nodes. Therefore, the system used Wi-Fi networks that could physically expand the network through the router nodes and seamlessly connect distributed nodes. In addition, two types of Wi-Fi networks were built in physically independent spaces by dividing them into gateways integrated with broker servers [7].

The sensor node is an and device that forms an MQTT client and Wi-Fi mesh network, which is connected to a gateway to transmit sensor data or perform a Public/subscribe function to control the actuator. Depending on the functions performed in this system, the Sensor Node consists of three types of nodes that perform all the functions of the Public communication function, the Subscribe communication function, and the Public and Subscribe. The MQTT protocol basically supports multiple MQTT gateways in a single wireless sensor network. Thus, the gateway consists of routers in the Wi-Fi mesh network, serves as a connection between the external Internet network and the Wi-Fi-based wireless network, and is tasked with converting and delivering messages to and from the format of the MQTT protocol [6].

The CoAP protocol client program was developed using the CoAP-Simple-library library's <coap.h>. The CoAP protocol is a protocol that operates on a UDP basis, and CoAP can establish reliability and non-reliability transmission among data types.

CoAP's Sub Gateway communicates wirelessly with the CoAP sensors located in each specific space. Sub Gateway is configured for each CoAP server and serves as the entrance for each CoAP server. Messages from the HTTP server are received and processed and forwarded to each CoAP sensor. Receive messages generated by the HTTP request creator and process them in the CoAP Proxy. The first thing to deal with is the proxy processing. The request message generated is separated by the host address and destination address, with the copy target uri query language in the middle. The host address will be temporarily stored in 
the proxy to transmit response messages from the destination address, and the destination address will handle the queries described by the user. The following steps are the process of processing queries, which consists of navigating objects, creating multicast groups, multicasting, and processing responses.

To check the amount of electricity used for each low-power Internet of Things protocol, the analysis is based on condensation values measured by the system.

Basic power consumption was measured with the experimental ATmega318 powered by the command interface of the Arduino IDE and no work except the basic process and fine dust sensor. It is connected to an external Wi-Fi router by installing a Wi-Fi module in the Arduino Uno [10].

With Wi-Fi module installed, it was able to confirm that average electricity consumed by using average power and general Wi-Fi signals that transmit fine dust sensor data is consumed by about $1,520 \mathrm{~mW}$. For the basic power consumption of $1520 \mathrm{~mW}$, it is about $304 \mathrm{~mA}$ when converted from $5 \mathrm{~V}$ to current consumption. This is a power consumption equivalent to 33 hours of post-buffering, if the auxiliary battery with a capacity of $10000 \mathrm{mAh}$ is used. Currently, Arduino does not support low power mode (slip mode), which shows relatively large electricity consumption. This is judged to be used in an environment where external Wi-Fi modules are used, increasing the amount of power consumed. Taking this into account, the comparison analysis was conducted by measuring the amount of power consumed by each communication protocol, and five minutes per time was tested using each communication protocol, and the average power generated by each of the five tests of each communication protocol was calculated and compared.

Based on the amount of power consumed by the most used HTTP communication protocol in the Wi-Fi environment, we compared the amount of power consumed by the MQTT and CoAP communication protocols.

In addition to the amount of power consumed by MQTT and CoAP over a period of 1 to 5 seconds, and the amount of power consumed by as well as the message/response supported by QoS and CoAP supported by MQTT.

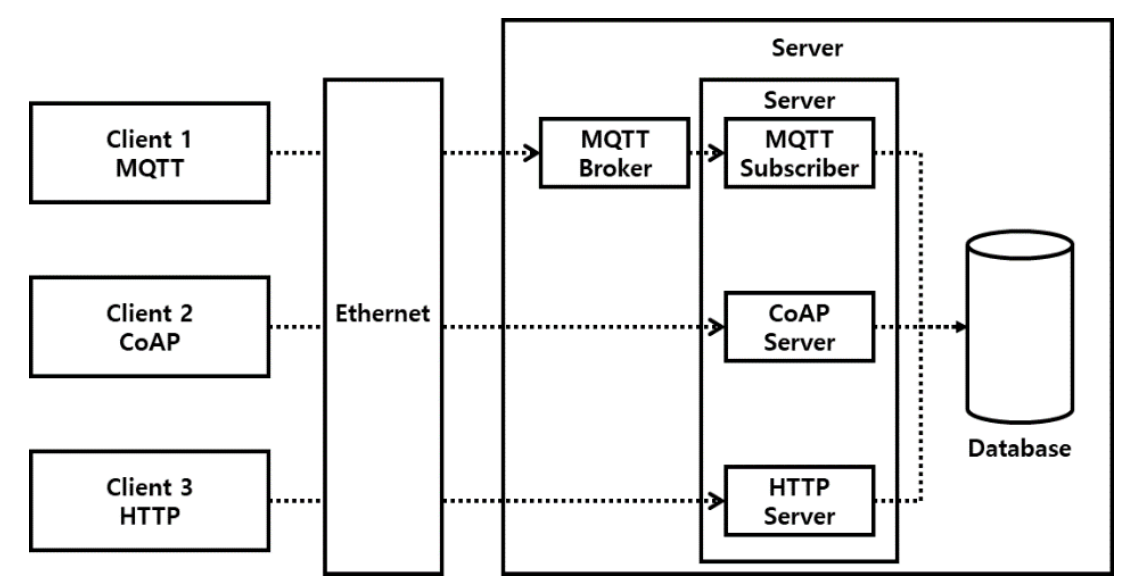

Figure 1. Test system configuration

\section{Results and analysis}

Tests using the HTTP protocol show the power consumed by each test. 
[Figure 2] shows the results of measuring the amount of power consumed and is applied to other protocol tests with the same measurement conditions for HTTP.

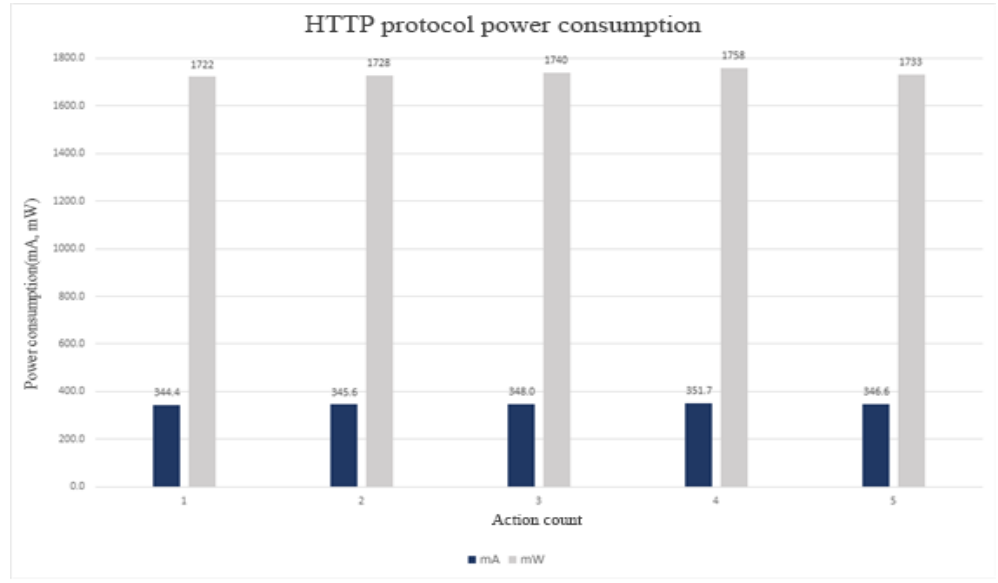

Figure 2. HTTP protocol power consumption

For the HTTP protocol, the current will burn from a minimum of $344.4 \mathrm{~mA}$ to a maximum of $351.7 \mathrm{~mA}$, and from a minimum of $1,722 \mathrm{~mW}$ to a maximum of $1758 \mathrm{~mW}$ for power.

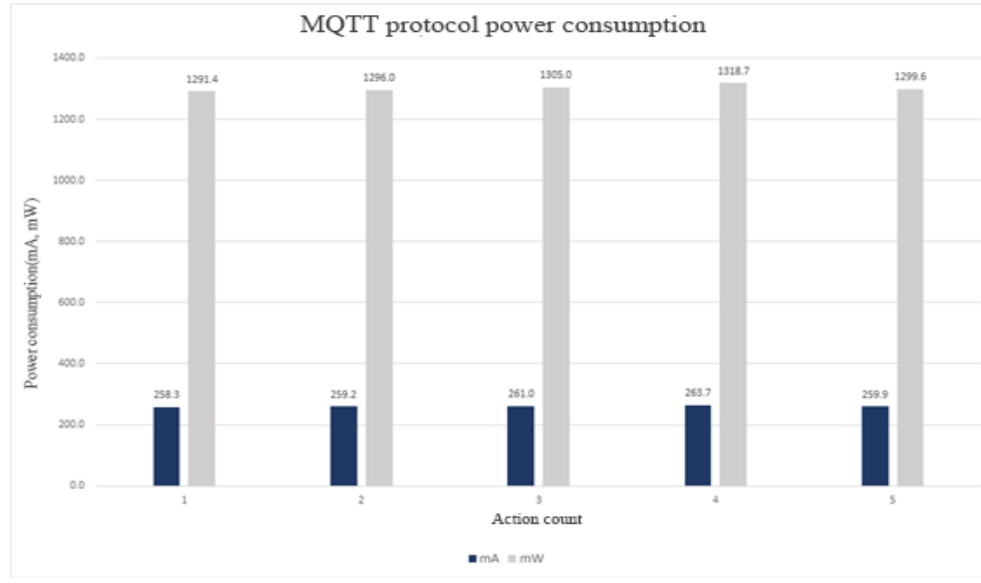

Figure 3. MQTT protocol power consumption

Tests using the MQTT protocol show the power consumed by each test.

[Figure 3] shows the results of the measurement of power consumption and was used in the test environment under the same conditions as HTTP. The current will burn from a minimum of $258.3 \mathrm{~mA}$ to a maximum of $263.7 \mathrm{~mA}$, and from a minimum of $1,291 \mathrm{~mW}$ to $1,318.5 \mathrm{~mW}$ for power.

Tests using the CoAP protocol show the power consumed by each test.

[Figure 4] shows the results of the measurement of power consumption and was conducted in the test environment under the same conditions as HTTP to measure the amount of power consumed. The current will burn from a minimum of $290.5 \mathrm{~mA}$ to $295.5 \mathrm{~mA}$, and from a minimum of $1424.4 \mathrm{~mW}$ to $1447.8 \mathrm{~mW}$ for power. 


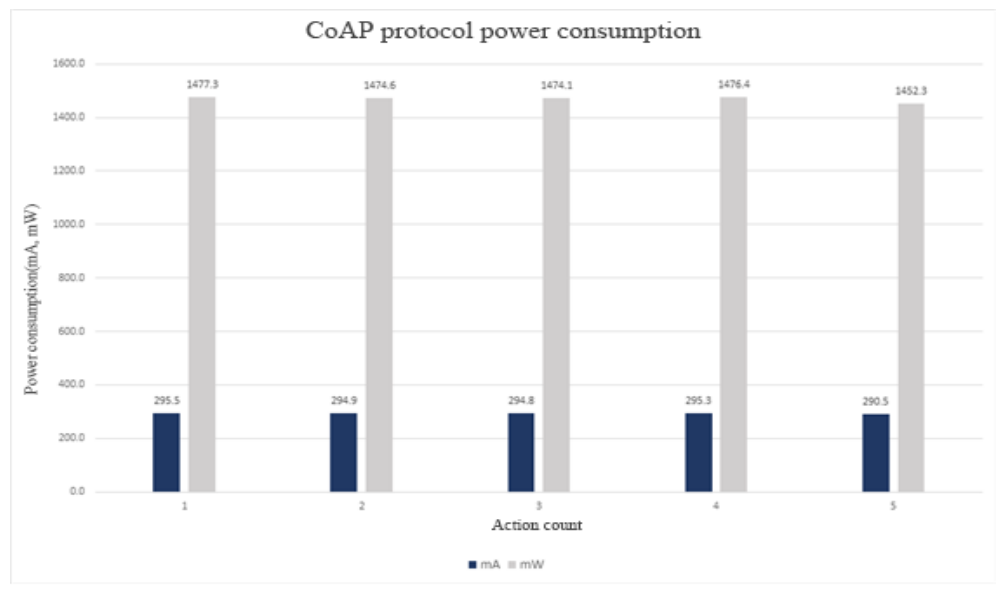

Figure 4. CoAP protocol power consumption

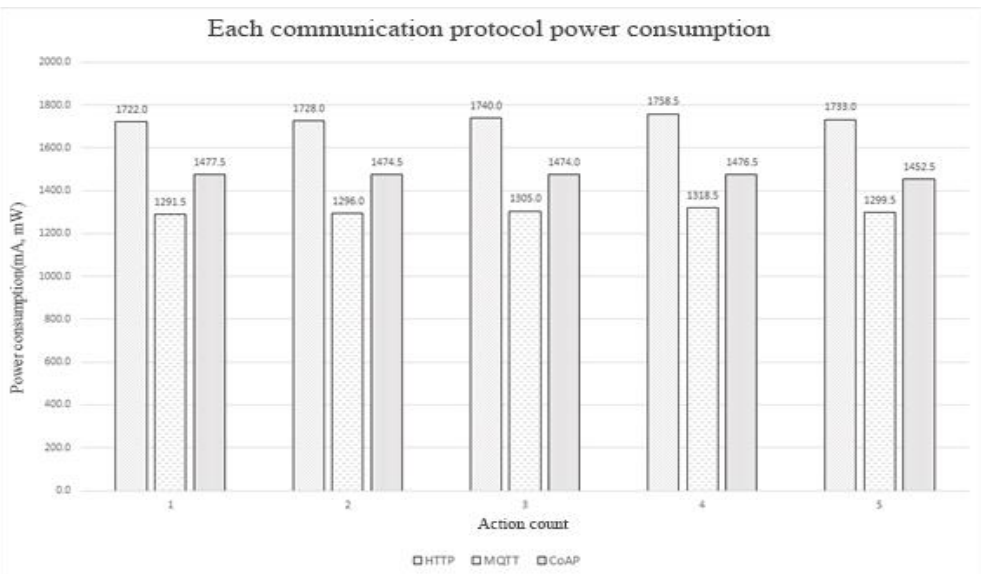

Figure 5. Each communication protocol power consumption

For [Figure 5], the overall comparison of each protocol shows low power consumption in MQTT's protocols. The MQTT protocol shows about $28 \%$ power efficiency and the CoAP protocol shows about $25 \%$ power efficiency over the existing HTTP communication protocol.

Recently, services and objects using the Internet of Things have appeared in Korea as well as abroad. With the number of Internet of Things devices expected to continue to increase, light-weight protocols that can be used efficiently by devices with limited resources such as sensor devices are also gaining traction.

This paper carried out an experiment focusing on fine dust data that should be continuously measured and compared and analyzed the suitability of any of the lightened protocols.

\section{Conclusion}

For the HTTP protocol, it will consume at least $344.4 \mathrm{~mA}$ to up to $351.7 \mathrm{~mA}$, and for power, it will consume at least $1,722 \mathrm{~mW}$ to $1758 \mathrm{~mW}$. The MQTT protocol will consume power from a minimum of $258.3 \mathrm{~mA}$ to a maximum of $263.7 \mathrm{~mA}$, and from a minimum of $1,291 \mathrm{~mW}$ to $1318.5 \mathrm{~mW}$ for power. The CoAP protocol will consume at least $290.5 \mathrm{~mA}$ to $295.5 \mathrm{~mA}$, and 
at least $1424.4 \mathrm{~mW}$ to $1447.8 \mathrm{~mW}$ for power. The overall comparison of each protocol shows low power consumption in MQTT's protocols. The MQTT protocol shows about 28\% power efficiency and the CoAP protocol shows about $25 \%$ power efficiency over the existing HTTP communication protocol.

In environments such as sensing data that simply transmits measured data, such as ambient atmospheric environments, the MQTT message type was shown to provide better results than previously used HTTP communication protocols in environments where the use of MQTT message types is appropriate and cannot reliably power, and where consumption should be prioritized if long hours of availability should be guaranteed with low power.

\section{Acknowledgements}

This study was supported by a 2019 research grant from Hanseo University and was supported by the Korea Institute for Advancement of Technology (KIAT) grant funded by the Korea government (MOTIE: Ministry of Trade, Industry \& Energy) (No. N0002431).

\section{References}

[1] D. Cearley, Top 10 Strategic Technology Trends for 2016, Gartner / Symposium ITxpo 2015, October 4-8, Orlando American, (2015)

[2] S. Y. Kim and K.Y. Kim, "Standardization of IoT," Service Platform, vol.6, no.32, (2014)

[3] oneM2M, oneM2M Service Layer Protocol Core Specification, oneM2M, (2014)

[4] Milan Tucic, "Networking layer for unifying distributed smart home entities," 22nd Telecommunications forum TELFORNovember 25-27, Belgrade, Serbia, (2014)

[5] Thread Group, "Network architecture," Micro Embedded Conference, (2014)

[6] M. Prihodko, "Energy consumption in location sharing protocols for Android applications," M.S. thesis, Linköopings University, (2012)

[7] J. S. Myeong, "IoT Service Platform Standardization Status,” TTA Journal, vol.166, pp.25-29, (2016)

[8] M. Dong, T. Lan, and L. Zhong, "Rethink energy accounting with cooperative game theory," MobiCom '14: Proceedings of the 20th annual international conference on Mobile computing and networking, September 531-542, New York, United States, (2014)

[9] X. Liu and F. Qian, "Poster: Measuring and Optimizing Android Smartwatch Energy Consumption," Proceedings of the 22nd Annual International Conference on Mobile Computing and Networking, pp.421-423, (2016)

[10] Message Queuing Telemetry Transport, (2014)

\section{Authors}

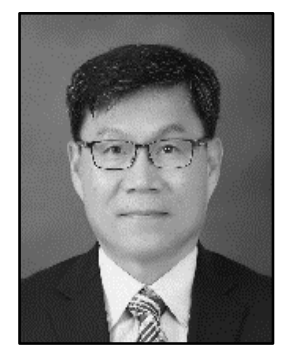

\section{Seongse Cho}

Received his bachelor's degree in computer science from the Korea National Open University in 2003. In 2017, he received the Master degree in avionics engineering from Hanseo University, Seosan, Korea. His research interests include military fighter jet avionics system, UAV collision avoidance, robotics, and intelligent transportation systems. 


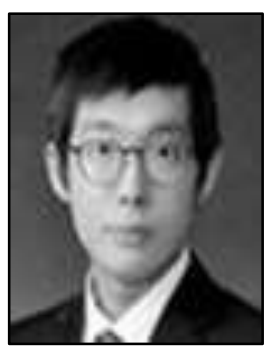

\section{Seung-Hun Kim}

He is a master's degree in Aeronautical System Engineering, Hanseo University. He graduated from the Department of Aeronautical and Mechanical Engineering, Hanseo University. He is studying Kalman filters, embedded systems, and smart network communications.

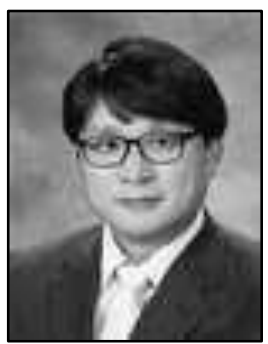

\section{Won-Hyuck Choi}

He received the Ph.D. degree in avionics from Korea Aerospace University, Korea. He was a Professor of smart network at Doowon Technical University College, Korea. urrently, he is Professor of avionics at Hanseo University, Korea, where he has been since 2014. His present research interests include embedded system, home network. 
Comparison of Communication Protocol Power Consumption of Fine-Dust loT Sensor Based on oneM2M Standard Protocol

This page is empty by intention. 